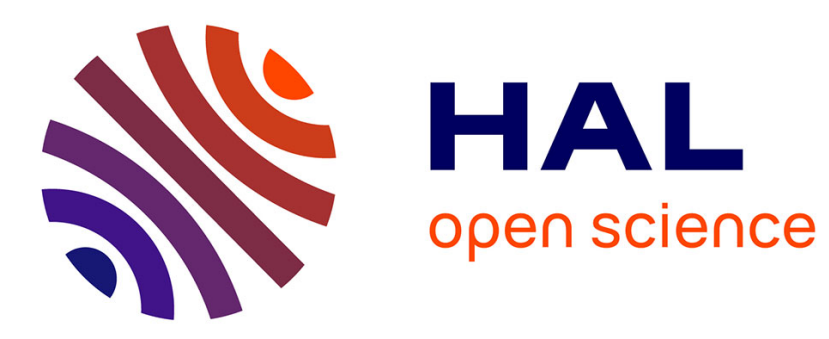

\title{
Doppler cooling of calcium ions using a dipole-forbidden optical transition
}

\author{
Richard J. Hendricks, Jens L. Sørensen, Caroline Champenois, Martina \\ Knoop, Michael Drewsen
}

\section{To cite this version:}

Richard J. Hendricks, Jens L. Sørensen, Caroline Champenois, Martina Knoop, Michael Drewsen. Doppler cooling of calcium ions using a dipole-forbidden optical transition. Physical Review A : Atomic, molecular, and optical physics [1990-2015], 2008, 77 (2), pp.021401(R). 10.1103/PhysRevA.77.021401 . hal-00979372

\section{HAL Id: hal-00979372 https://hal.science/hal-00979372}

Submitted on 15 Apr 2014

HAL is a multi-disciplinary open access archive for the deposit and dissemination of scientific research documents, whether they are published or not. The documents may come from teaching and research institutions in France or abroad, or from public or private research centers.
L'archive ouverte pluridisciplinaire HAL, est destinée au dépôt et à la diffusion de documents scientifiques de niveau recherche, publiés ou non, émanant des établissements d'enseignement et de recherche français ou étrangers, des laboratoires publics ou privés. 


\title{
Doppler cooling of calcium ions using a dipole-forbidden transition
}

\author{
Richard J. Hendricks, ${ }^{1}$ Jens L. Sørensen, ${ }^{1}$ Caroline Champenois, ${ }^{2}$ Martina Knoop, ${ }^{2}$ and Michael Drewsen ${ }^{1, *}$ \\ ${ }^{1}$ QUANTOP_Danish National Research Foundation Center for Quantum Optics, Department of Physics and Astronomy, \\ University of Aarhus, DK-8000 Århus C, Denmark \\ ${ }^{2}$ Physique des Interactions Ioniques et Moléculaires (CNRS UMR 6633), Université de Provence, Centre de Saint Jérôme, \\ Case C21, 13397 Marseille Cedex 20, France
}

(Received 12 July 2007; published 4 February 2008)

\begin{abstract}
Doppler cooling of calcium ions has been experimentally demonstrated using the $S_{1 / 2} \rightarrow D_{5 / 2}$ dipoleforbidden transition. Scattering forces and fluorescence levels a factor of 5 smaller than for the usual Doppler cooling on the dipole-allowed $S_{1 / 2} \rightarrow P_{1 / 2}$ transition have been achieved. Since the light scattered from the ions can be monitored at (violet) wavelengths that are very different from the excitation wavelengths, single ions can be detected with an essentially zero background level. This, as well as other features of the cooling scheme, can be extremely valuable for ion-trap-based quantum-information processing.
\end{abstract}

DOI: 10.1103/PhysRevA.77.021401

PACS number(s): 37.10.De, 37.10.Gh

In the last few decades, a large variety of laser cooling techniques have been proposed and demonstrated [1,2]. These include Doppler cooling [3,4], sideband cooling [5-8], stimulated cooling [9], polarization gradient cooling [10-12], magnetically induced laser cooling [13], Raman cooling $[14,15]$, and intercombination line cooling $[16,17]$. A characteristic of these cooling schemes is a trade-off between the velocity range where the cooling mechanism is efficient and the final achievable minimum temperature. As a consequence, most experiments today use more than one cooling mechanism. For quasifree neutral atoms with degenerate ground state sublevels, a combination of Doppler and polarization gradient cooling can be used relatively easily to obtain dense ensembles of atoms in the microkelvin range $[10,18]$. For neutral atoms lacking ground state sublevels, such as most alkaline-earth-metal elements, a more elaborate cooling scheme such as a second Doppler cooling stage on a weak intercombination line is required in order to obtain ultracold atoms $[16,17,19]$. With respect to trapped ions, a two-step cooling process consisting of a Doppler cooling phase followed by a resolved-sideband cooling phase is typically used to cool from initial high thermal energies to close to the ground state of the trapping potential. For the sideband cooling step, either a Raman cooling scheme [7], an electromagnetically induced transparency cooling process [8], or excitation on a weak, narrow-linewidth transition $[6,20]$ is used.

In this Rapid Communication, we demonstrate a scheme for Doppler cooling of ${ }^{40} \mathrm{Ca}^{+}$ions that relies on the combined action of driving the $S_{1 / 2} \rightarrow D_{5 / 2}$ electric quadrupole transition and the dipole-allowed $D_{5 / 2} \rightarrow P_{3 / 2}$ transition [Fig. 1(a)]. While the required velocity dependence of the cooling force is obtained through excitation of the $S_{1 / 2} \rightarrow D_{5 / 2}$ quadrupole transition by an intense laser beam at $729 \mathrm{~nm}$, a copropagating $854 \mathrm{~nm}$ "assisting" laser beam is applied to drive the $D_{5 / 2} \rightarrow P_{3 / 2}$ dipole transition. This latter laser field increases the effective decay rate $\Gamma^{\prime}$ of the $D_{5 / 2}$ state to the $S_{1 / 2}$ ground state, as well as increasing the cooling force because of the

\footnotetext{
*drewsen@phys.au.dk
}

extra momentum transferred to the ions by the $854 \mathrm{~nm}$ photons. Ground state cooling using a resolved sideband of the $S_{1 / 2} \rightarrow D_{5 / 2}$ quadrupole transition has already been demonstrated in the regime where $\Gamma^{\prime}$ is smaller than the relevant motional mode frequency of the trapped ions $\omega$ [20]. In contrast to this, we here consider Doppler cooling, where $\Gamma^{\prime}>\omega$. By using a narrow-bandwidth $729 \mathrm{~nm}$ laser, lowering the intensities, and changing the detunings of the lasers the presented cooling scheme can, however, be transformed into a resolved-sideband cooling scheme with little additional complexity. In our experiments, scattering forces and fluorescence levels only about a factor of 5 smaller than for the usual Doppler cooling on the dipole-allowed $S_{1 / 2} \rightarrow P_{1 / 2}$ transition have been achieved. As discussed at the end of this Rapid Communication, the Doppler cooling scheme presented here has several features of particular interest for quantum-information processing with trapped ions.

A reduced level scheme of $\mathrm{Ca}^{+}$is shown in Fig. 1(a), together with the laser excitations and fluorescence paths of interest for the presented cooling scheme. The key laserexcited transitions in the cooling scheme are the $S_{1 / 2} \rightarrow D_{5 / 2}$

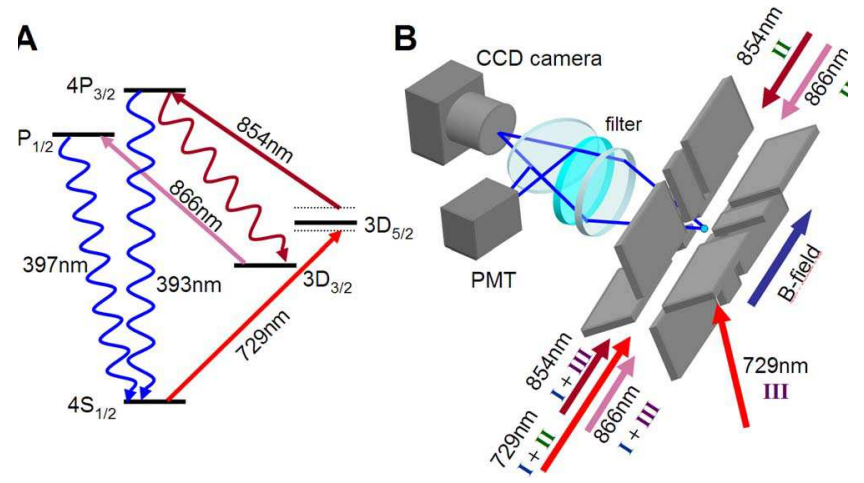

FIG. 1. (Color online) (a) Relevant energy levels and transitions of the ${ }^{40} \mathrm{Ca}^{+}$ion. (b) Experimental setup. Three different situations are illustrated, I: Axial cooling where the cooling and assisting lasers are copropagating. II: Axial cooling with counterpropagating cooling and assisting lasers. III: Three-dimensional cooling with the cooling laser at an angle of $45^{\circ}$ to the trap axis and the assisting laser parallel to the trap axis. 
electric quadrupole transition at $729 \mathrm{~nm}$, which has a natural width of just $\Gamma_{c}=2 \pi \times 0.14 \mathrm{~Hz}$ [21], and the $854 \mathrm{~nm}$ $D_{5 / 2} \rightarrow P_{3 / 2}$ dipole transition, which has a linewidth of $\Gamma=2 \pi \times 23 \mathrm{MHz}$. Ions excited to the $P_{3 / 2}$ level will predominantly decay to the $S_{1 / 2}$ ground state by the emission of light at $393 \mathrm{~nm}$. They can also, however, decay with probabilities 0.07 and 0.008 to the $D_{5 / 2}$ and $D_{3 / 2}$ levels, respectively, emitting infrared light. To prevent optical pumping into the $D_{3 / 2}$ level, a repumper laser at $866 \mathrm{~nm}$ is used to excite ions to the $P_{1 / 2}$ level, from where the ground state can be reached via the emission of a $397 \mathrm{~nm}$ photon.

The experimental setup is sketched in Fig. 1(b). The ${ }^{40} \mathrm{Ca}^{+}$ ions are stored in a segmented linear radio-frequency trap (details of which can be found in [22]) housed in an ultrahigh-vacuum chamber with a residual pressure of approximately $6 \times 10^{-10} \mathrm{mbar}$. The ions are loaded into the trap via isotope-selective photoionization [23], and the trap parameters are set such that the single-ion axial and radial motional frequencies are approximately $2 \pi \times 0.56 \mathrm{MHz}$ and $2 \pi \times 0.95 \mathrm{MHz}$, respectively. Sets of Helmholtz coils are used to null the magnetic field to a level of about $5 \mathrm{mG}$.

The $729 \mathrm{~nm}$ light, which has a linewidth of approximately $200 \mathrm{kHz}$, is generated by a Ti:sapphire laser stabilized to an external optical cavity. Up to $250 \mathrm{~mW}$ of power is available in a beam focused to a waist of $50 \mu \mathrm{m}$ at the trap center. This leads to a maximum achievable quadrupole transition Rabi frequency of $\sim 2 \pi \times 1 \mathrm{MHz}$ [24]. The beam propagates either parallel to or at an angle of $45^{\circ}$ to the axis of the trap, with the latter configuration enabling cooling of both the axial and radial motion of the trapped ions. The light is always linearly polarized within the horizontal plane. The $854 \mathrm{~nm}$ assisting and the $866 \mathrm{~nm}$ repumper laser beams are derived from extended-cavity diode lasers locked to external optical cavities and have linewidths of about $300 \mathrm{kHz}$. A maximum of $3 \mathrm{~mW}$ is available at each wavelength, and beam waists of $\sim 280 \mu \mathrm{m}$ are used. Both beams can either co- or counterpropagate with the $729 \mathrm{~nm}$ laser beam when the latter is directed along the axis of the trap. The polarization of both these lasers is rotated at a frequency of $4 \mathrm{MHz}$ using an electro-optic modulator to prevent the formation of "dark" states [25]. In order to keep the ac Stark shift and broadening of the $D_{5 / 2}$ level Doppler insensitive at low ion velocities, the $854 \mathrm{~nm}$ laser is kept at a constant detuning of approximately $100 \mathrm{MHz}$. With a power of $1 \mathrm{~mW}$ this leads to an effective decay rate of the $D_{5 / 2}$ level, $\Gamma^{\prime}$, of about $2 \pi$ $\times 2 \mathrm{MHz}$, which is larger than the single-ion oscillation frequencies.

Since the laser power at $729 \mathrm{~nm}$ is limited and the overlap between the trapping volume and the $729 \mathrm{~nm}$ laser beam is small, cooling of the ions using the 729 and $854 \mathrm{~nm}$ lasers is not very efficient in the phase immediately after loading, where the ions have kinetic energies in the $\mathrm{eV}$ range. For this reason, in all experiments the ions are first Doppler cooled in all directions using the $S_{1 / 2} \rightarrow P_{1 / 2}$ dipole-allowed transition at $397 \mathrm{~nm}$ in the presence of the $866 \mathrm{~nm}$ repumper. Mechanical shutters with a switching time of approximately $0.1 \mathrm{~ms}$ are used to change between the 397 and $729 \mathrm{~nm}$ cooling configurations.

Ions are detected by imaging the 393 and $397 \mathrm{~nm}$ fluorescence light onto both a photomultiplier-tube-based photon-

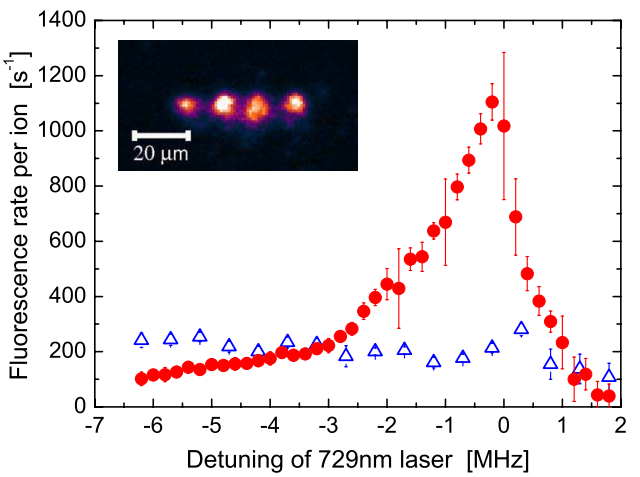

FIG. 2. (Color online) Detected fluorescence rate from four ions as a function of the $729 \mathrm{~nm}$ laser detuning for copropagating (red, filled circles) and counterpropagating (blue, open triangles) cooling and assisting laser beams. The inset shows a CCD image of the four ions.

counting module and an image-intensified charge-coupled device (CCD) camera. Filters are used to prevent any light at red or infrared wavelengths from reaching the detectors. The overall detection efficiencies of the photon-counting module and the CCD camera system are $3.6 \times 10^{-4}$ and $8.4 \times 10^{-5}$, respectively.

In Fig. 2, the detected fluorescence rate from a string of four ions is presented as a function of the $729 \mathrm{~nm}$ cooling laser detuning. Each plotted point is an average of 20 consecutive measurements, with the error bars representing the standard deviation of these. Each individual measurement is preceded by precooling on the dipole-allowed transition, and the fluorescence level is measured over $200 \mathrm{~ms}$ after these precooling beams are switched off. For all data points the $729 \mathrm{~nm}$ laser beam propagates along the axis of the trap, with the assisting laser beam being either co- or counterpropagating. For the counterpropagating case, the fluorescence level does not change significantly with the detuning, indicating that there is little change in the velocity distribution of the ions. In the copropagating scenario, however, the fluorescence signal shows a pronounced resonant structure indicating a strong mechanical effect of the light fields. This striking difference, which is not pronounced in resolved sideband cooling, can be explained by the fact that the twophoton momentum kick received by the ion when excited to the $P_{3 / 2}$ level is about 13 times smaller in the counterpropagating situation than in the case of copropagating beams. At the largest negative detunings, the fluorescence rate for copropagating beams is clearly lower than for counterpropagating beams, indicating that the ions are so efficiently cooled that they Doppler tune out of resonance.

Another way to characterize the cooling effect is to monitor the change in position (jumping) of a single sympathetically cooled ion in an ion string over time. Only if the kinetic energy of the ions is high enough to overcome the rearrangement potential barrier, typically corresponding to $\sim 1 \mathrm{~K}$, will a jump take place. In Fig. 3, the fraction of times $R$ that a single, nonfluorescing, sympathetically cooled ion in a fourion string is observed to change position during a $200 \mathrm{~ms}$ period of cooling is presented as a function of the detuning of the $729 \mathrm{~nm}$ laser. The corresponding integrated fluores- 


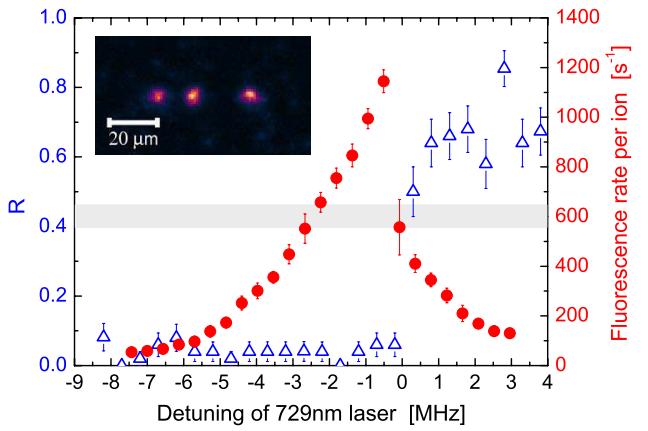

FIG. 3. (Color online) Fraction of times, $R$, that a nonfluorescing ion in an ion string is seen to switch position as a function of $729 \mathrm{~nm}$ laser detuning (blue, open triangles), along with the corresponding fluorescence rate (red, filled circles). The average jump rate without the $729 \mathrm{~nm}$ laser is also shown (shaded horizontal bar). The inset shows a CCD image of the four-ion string, with the gap indicating the position of the nonfluorescing ion.

cence is also plotted. Clearly there is a dramatic change in the parameter $R$ around zero detuning, which indicates that cooling is indeed taking place at all negative detunings and heating at positive detunings. This conclusion is further supported by comparison with the value of $R$ when no cooling beams are present. The nonzero value of $R$ in this case is mainly due to trap-induced heating [26]. Since the resonance of the $729 \mathrm{~nm}$ cooling transition is shifted from that of the unperturbed transition by the presence of the $854 \mathrm{~nm}$ assisting laser, we have defined the zero detuning in Figs. 2 and 3 to be the frequency at which $R$ starts to increase steeply.

Based on the observed fluorescence rates and the efficiencies of the photon-detecting devices, the maximum achieved scattering forces in the co- and counterpropagating configurations are estimated to be $4.2 \times 10^{-21}$ and $3.3 \times 10^{-22} \mathrm{~N}$, respectively. While the latter is nearly two orders of magnitude smaller than for standard Doppler cooling using the $S_{1 / 2} \rightarrow P_{1 / 2}$ dipole-allowed transition, the copropagating geometry gives a force that is only about a factor of 5 lower. The widths of the resonances in the fluorescence are limited by several effects, but assuming Doppler broadening due to the finite temperature of the ions to be dominant, a conservative estimate of the ion temperature of a few millikelvin is obtained.

With the $729 \mathrm{~nm}$ cooling laser propagating at an angle of $45^{\circ}$ to the axis of the trap, both axial and radial motions of the ions have been cooled. Using such a configuration, single ions have remained laser cooled for periods of up to a few minutes. This time seems to be limited by a nonsufficient velocity capture range with the present power level of the $729 \mathrm{~nm}$ laser to recool ions that have collided with background atoms or molecules.

A simple way to overcome this problem would be to increase the velocity capture range by increasing the power of the $729 \mathrm{~nm}$ laser. At this wavelength, narrow-bandwidth lasers with output powers of several watts have been demonstrated [27]. A complementary path to enlarge the velocity capture range is to exploit the fact that the $S_{1 / 2} \rightarrow D_{5 / 2}$ transition actually consists of ten Zeeman components, which can be split by applying a magnetic field. For a negative

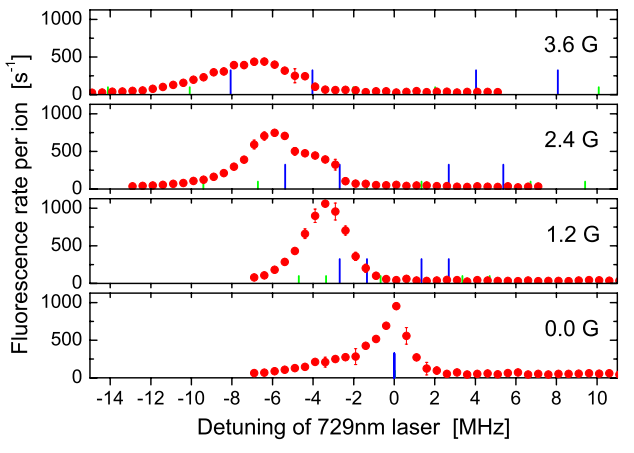

FIG. 4. (Color online) Detected fluorescence as a function of $729 \mathrm{~nm}$ laser detuning for different magnetic field strengths. The vertical blue bars indicate the positions of the four $\Delta m_{j}= \pm 1$ Zeeman components to which the laser couples.

detuning of the $729 \mathrm{~nm}$ laser with respect to all the Zeemansplit transitions, a cooling force with a maximum value at different velocities will exist for each of the Zeeman lines. In Fig. 4, a series of fluorescence spectra is presented for cooling experiments in the axial cooling geometry with various magnetic field strengths applied parallel to the trap axis. In this geometry only the four $\Delta m_{j}= \pm 1$ lines are active [24]. As the magnetic field is increased from zero to $1.2 \mathrm{G}$, a significant broadening is observed without any drop in maximum fluorescence. At higher magnetic fields the Zeeman splitting of the lines becomes comparable to the width of the power-broadened forbidden transition, leading to optical pumping into the more weakly coupled Zeeman substate. This results in a lower scattering rate, which is visible in the upper two panels of Fig. 4.

The presented Doppler cooling scheme could be made slightly simpler by exciting the $733 \mathrm{~nm} S_{1 / 2} \rightarrow D_{3 / 2}$ transition instead of the $S_{1 / 2} \rightarrow D_{5 / 2}$ transition. In this case the $866 \mathrm{~nm}$ laser acts as the assisting laser without need for any repumper laser. In quantum logic (metrology) experiments where $S_{1 / 2}$ and $D_{5 / 2}$ substates are the qubit (relevant) states this scheme would be favorable, since the two cooling lasers can also be used in projection measurements $[20,28]$.

Independent of which transition is used, the benefits of the presented Doppler cooling scheme are manifold. First, it reduces the number of lasers and laser beams needed to eventually obtain ground state cooling. Only changes to the intensities and frequencies of the two cooling lasers are needed to meet the conditions for resolved-sideband cooling previously demonstrated on the $S_{1 / 2} \rightarrow D_{5 / 2}$ quadrupole transition [20]. Second, light scattered from the ions is at violet wavelengths that are very different from the red and infrared excitation wavelengths. This is equivalent to the situation in two-photon microscopy [29], and leads to an essentially zero spurious scattering background level. This feature can be extremely valuable for fast readout of qubits in ion-trap quantum-information processing [30,31]. Third, the requirement that the 729 or $733 \mathrm{~nm}$ laser have a high intensity and consequently small focus is very compatible with modern microtraps, in which tight laser focuses are in any case required. These traps have typical electrode spacings of $\sim 100 \mu \mathrm{m}$ [32], and the presented cooling scheme benefits 
from the excellent overlap between the trapping volume and the laser beams that can be achieved. Fourth, only lasers in the red and infrared part of the spectrum, where narrowbandwidth high-power lasers are widely available, are applied. Such technological aspects may play an important role when deciding on a specific ion species for large-scale quantum-information processing [33]. Finally, in connection with sympathetic cooling of complex molecular ions, the problem of photofragmentation might in some cases be reduced by using red instead of violet light [34].

Although in the present investigations we have focused on the ${ }^{40} \mathrm{Ca}^{+}$ion, the cooling scheme can also be applied to the other isotopes of calcium as well as other ions with a similar atomic level structure, such as $\mathrm{Sr}^{+}, \mathrm{Ba}^{+}$, and $\mathrm{Hg}^{+}$[24]. For sympathetic cooling of complex molecules, $\mathrm{Ba}^{+}$is a particularly interesting ion since the $S_{1 / 2} \rightarrow D_{3 / 2}$ transition is at
$2051 \mathrm{~nm}$ - a wavelength at which large molecules often do not absorb and at which powerful commercial fiber lasers exist.

In conclusion, a Doppler cooling scheme relying on excitation of the $S_{1 / 2} \rightarrow D_{5 / 2}$ electric quadrupole transition in the ${ }^{40} \mathrm{Ca}^{+}$ion has been demonstrated. The cooling scheme, which is readily extendable to other alkaline-earth-ion species, contains several features that are interesting for iontrap-based quantum-information processing and cold complex molecular ion research.

This work is financially supported by the Carlsberg Foundation and by the EU under Contract No. IST-517675MICROTRAP. M.D. gratefully acknowledges the hospitality of the Laboratoire PIIM-CIML at the Universite de Provence under a visiting professor grant.
[1] C. Adams and E. Riis, Prog. Quantum Electron. 21, 1 (1997).

[2] D. Leibfried, R. Blatt, C. Monroe, and D. Wineland, Rev. Mod. Phys. 75, 281 (2003).

[3] J. V. Prodan, W. D. Phillips, and H. Metcalf, Phys. Rev. Lett. 49, 1149 (1982).

[4] S. Chu, L. Hollberg, J. E. Bjorkholm, A. Cable, and A. Ashkin, Phys. Rev. Lett. 55, 48 (1985).

[5] W. Neuhauser, M. Hohenstatt, P. Toschek, and H. Dehmelt, Phys. Rev. Lett. 41, 233 (1978).

[6] F. Diedrich, J. C. Bergquist, W. M. Itano, and D. J. Wineland, Phys. Rev. Lett. 62, 403 (1989).

[7] C. Monroe et al., Phys. Rev. Lett. 75, 4011 (1995).

[8] C. F. Roos et al., Phys. Rev. Lett. 85, 5547 (2000).

[9] A. Aspect, J. Dalibard, A. Heidmann, C. Salomon, and C. Cohen-Tannoudji, Phys. Rev. Lett. 57, 1688 (1986).

[10] P. D. Lett, R. N. Watts, C. I. Westbrook, W. D. Phillips, P. L. Gould, and H. J. Metcalf, Phys. Rev. Lett. 61, 169 (1988).

[11] J. Dalibard and C. Cohen-Tannoudji, J. Opt. Soc. Am. B 6, 2023 (1989).

[12] P. J. Ungar et al., J. Opt. Soc. Am. B 6, 2058 (1989).

[13] S.-Q. Shang, B. Sheehy, P. van der Straten, and H. Metcalf, Phys. Rev. Lett. 65, 317 (1990).

[14] M. Kasevich and S. Chu, Phys. Rev. Lett. 69, 1741 (1992).

[15] J. Reichel et al., Phys. Rev. Lett. 75, 4575 (1995).

[16] T. Binnewies et al., Phys. Rev. Lett. 87, 123002 (2001).
[17] E. A. Curtis, C. W. Oates, and L. Hollberg, Phys. Rev. A 64, 031403(R) (2001).

[18] C. Salomon et al., Europhys. Lett. 12, 683 (1990).

[19] T. Mukaiyama, H. Katori, T. Ido, Y. Li, and M. KuwataGonokami, Phys. Rev. Lett. 90, 113002 (2003).

[20] C. F. Roos et al., Phys. Rev. Lett. 83, 4713 (1999).

[21] P. A. Barton et al., Phys. Rev. A 62, 032503 (2000).

[22] J. L. Sørensen et al., New J. Phys. 8, 261 (2006).

[23] N. Kjærgaard et al., Appl. Phys. B: Lasers Opt. 71, 207 (2000).

[24] D. F. V. James, Appl. Phys. B: Lasers Opt. 66, 181 (1998).

[25] G. P. Barwood et al., Opt. Commun. 151, 50 (1998).

[26] L. Deslauriers et al., Phys. Rev. A 70, 043408 (2004).

[27] H. Müller et al., Opt. Lett. 31, 202 (2006).

[28] C. F. Roos et al., Nature (London) 443, 316 (2006).

[29] W. Denk, J. H. Strickler, and W. W. Webb, Science 248, 73 (1990).

[30] D. Leibfried et al., Nature (London) 438, 639 (2005).

[31] H. Häffner et al., Nature (London) 438, 643 (2005).

[32] D. Stick et al., Nat. Phys. 2, 36 (2005).

[33] D. Kielpinski, C. Monroe, and D. J. Wineland, Nature (London) 417, 709 (2002).

[34] K. Kato and K. Yamanouchi, Chem. Phys. Lett. 397, 237 (2004). 